\title{
An Evidence-Based Assessment Tool for Estimating Future Post-Traumatic Stress Disorder: A 7-Year Follow-Up Study
}

\author{
Judith McFarlane, DrPH, ${ }^{1}$ John Maddoux, $\mathrm{PhD},{ }^{2}$ René Paulson, $\mathrm{PhD},{ }^{2}$ \\ Lene Symes, PhD, and Ernest N. Jouriles, $\mathrm{PhD}^{3}$
}

\begin{abstract}
Background: Intimate partner violence (IPV) affects up to one in three women across the world. Post-traumatic stress disorder (PTSD) is a common outcome. Many, but not all, women suffer long after they first sought help for IPV. Validated tools for estimating the likelihood of future PTSD are lacking.

Materials and Methods: Women who sought IPV support services for the first time in 2011-2012 $(N=300)$ completed a seven-item screen for the presence or absence of clinically significant PTSD symptoms and the first assessment screening tool for post-traumatic stress disorder (FAST-PTSD), a tool designed to estimate future PTSD among women seeking help for IPV. Seven years later, in 2018, 271 (90\%) women again completed the seven-item screen for clinically significant PTSD symptoms. A two-step binary logistic regression was conducted to determine the 7-year validity of the FAST for clinically significant symptoms of PTSD while controlling for baseline PTSD symptoms.

Results: More than 25\% of the women reported clinically significant PTSD at 7 years. Baseline moderate- and high-risk scores on the FAST-PTSD were associated with clinically significant levels of PTSD. Moderate risk was associated with nearly two and one-half times (odds ratio $[\mathrm{OR}]=2.4$ ) the risk of clinically significant symptoms of PTSD, and high risk with nearly eight times $(\mathrm{OR}=7.8)$ the risk of PTSD at 7 years.

Conclusions: PTSD is commonly associated with IPV and if untreated can compromise functioning of women and their children. The FAST-PTSD is a valid indicator of significant clinical PTSD symptoms 7 years following first contact with IPV support services. Using the FAST-PTSD to triage women at risk for sustained PTSD to early, preventive intervention may improve outcomes for women and their children.
\end{abstract}

Keywords: intimate partner violence, mental health, PTSD, screening

\section{Introduction}

NTIMATE PARTNER ViolenCe (IPV) is a worldwide problem affecting up to one in three women. ${ }^{1}$ Experiencing IPV can result in a wide range of mental health problems, with post-traumatic stress disorder (PTSD) among the most common mental health sequelae for women. ${ }^{2,3}$ PTSD influences women's day-to-day functioning and long-term health, and predicts the emotional and behavioral adjustment of their children. ${ }^{4,5}$ In short, PTSD is a common consequence of experiencing IPV for women, and it can have far-reaching negative effects. Although not all women who experience
IPV develop clinically significant PTSD symptoms, and such symptoms often abate over time, results from several prospective studies indicate that a sizable proportion of women suffer clinically significant PTSD symptoms months after they sought help for IPV. ${ }^{4,6,7}$ Women vulnerable to long-term PTSD following IPV are arguably in great need of clinical services when they first seek help for the IPV. ${ }^{8}$ Identifying such women early can aid efforts to improve their long-term health. ${ }^{9,10}$

The first assessment screening tool for post-traumatic stress disorder (FAST-PTSD) was developed to predict clinically significant PTSD symptoms among women seeking help for

${ }^{1}$ College of Nursing, Texas Woman's University, Houston, Texas.

${ }^{2}$ Elite Research, LLC, Irving, Texas.

${ }^{3}$ Department of Psychology, Southern Methodist University, Dallas, Texas. 
IPV, to identify those most at risk for long-term PTSD symptoms. ${ }^{11}$ The FAST-PTSD includes questions about current PTSD symptoms, but it also includes questions about adverse childhood experiences, emotional support, and general self-efficacy. It is more comprehensive than a simple screen for PTSD symptoms, but it is still easy and quick for service providers to administer.

In an initial study that described the development of the FAST-PTSD (referred to in the initial study as the rapid assessment tool to predict risk for sustained PTSD symptoms), women seeking services either at a domestic violence shelter or district attorney's office were recruited and followed for 16 months. ${ }^{11}$ FAST-PTSD scores were associated with women's PTSD symptoms at 8 and 16 months following their initial assessment. These findings on the potential utility of the FAST-PTSD are encouraging. However, the initial study did not evaluate whether FAST-PTSD scores were associated with later PTSD symptoms after accounting for baseline PTSD symptoms, and the follow-up period was limited to 16 months.

Building upon the findings of the initial study, the current research reports on 7-year follow-up data from the participants in the initial study. The first objective of this study was to document the prevalence of clinically significant PTSD symptoms 7 years after women first sought help. The second objective was to evaluate long-term associations between FAST-PTSD scores and PTSD symptoms. It was hypothesized that FAST-PTSD scores would be associated with clinically significant PTSD symptoms 7 years after women first sought help for IPV, after accounting for baseline PTSD symptoms.

\section{Materials and Methods}

Data for this study were baseline and final data obtained during a 7-year prospective study examining the mental and physical health outcomes of mothers and children, after mothers sought IPV-related support services, through either civil protection orders from the justice system or residential shelters for abused women, for the first time. Seven years was chosen by the funding agency to document long-term outcomes of PTSD for optimum program and policy decisions on resource allocation. At baseline (11/2010-06/2011), a total of 300 mother/child dyads were recruited based on a priori power analysis. At 7 years (01/2018-06/2018), 271 women provided data (retention rate: $90 \%$ ).

No significant differences were found between women who completed the study and those who were lost to followup. Data were collected in Spanish or English. The greatest percentage of the participants identified Spanish or Hispanic as their ethnic background $(n=137,45.7 \%)$, followed by black $(n=78,26.0 \%)$, white $(n=32,10.7 \%)$, and other $(n=20,6.6 \%)$. Other includes American Indian/Alaskan Native, Bi/multiracial, Asian. A full description of the demographics and metrics of the larger study can be found elsewhere. ${ }^{12}$ An institutional review board approved the current study. Signed informed consent was obtained from all participants.

\section{Measures}

The FAST-PTSD is a self-report questionnaire designed to predict future PTSD symptoms in women who report partner violence. Initial evaluation of the tool indicated a high degree of sensitivity and specificity at 8 and 16 months from initial contact, with greater than $90 \%$ accuracy when predicting positive PTSD symptoms at 8 months for individuals with high identified risk. The FAST-PTSD has four scales, with a total of 39 items, to assess adverse childhood experiences, current post-traumatic stress symptoms, social support, and self-efficacy. Following an algorithm, the results of the four scales are combined into a categorical score to indicate an individual's risk for chronic PTSD (no/minimal, low/some, moderate, high, or extreme risk). ${ }^{11}$

For this study, no/minimal and low/some risk categories are combined into no/minimal risk, moderate risk is retained, and high and extreme risk categories are combined into high risk. These categories were used to maximize group classification size and establish relatively equal frequencies across levels to increase the robustness of final models. These categories align with the triage recommendations across levels, with low and minimal risk indicating continued monitoring, and the moderate and higher levels indicating referral for additional services. The resulting three risk categories formed the predictor variable for this study.

The FAST-PTSD takes $\sim 10$ minutes to complete. For interested clinicians and researchers, the FAST-PTSD is available in English and Spanish as an electronic application for download from the World Wide Web, at no charge, onto Apple and Android devices.

The seven-item PTSD screen, developed by Breslau and Associates, has an established cutoff score of four or greater indicating the presence of clinically significant PTSD symptoms; lower scores indicate the absence of clinically significant PTSD symptoms. ${ }^{13}$ The seven-item screen yields a sensitive and specific diagnostic profile when compared with the current gold standard, Clinician-Administered PTSD Scale for DSM$5 .{ }^{14}$ The outcome variable for the current study was clinically significant PTSD symptoms (absent or present).

\section{Statistical analysis}

To examine the association between clinically significant levels of PTSD at 7 years from initial contact, a two-step binary logistic regression was conducted. Step 1 of the model assessed the association between clinically significant levels of PTSD at 7 years from initial PTSD symptoms, and step 2 examined the association between clinically significant levels of PTSD from FAST-PTSD scores (moderate and high risk) while controlling for baseline PTSD symptoms. No additional covariates were included in the model, as previously identified covariates were taken into account in the development of the FAST-PTSD tool; adding covariates into the model a subsequent time would create redundancy in the models.

Concerns of multicollinearity were examined with Pearson's product moment correlations, which suggested a moderate correlation $(r=0.74)$ between baseline PTSD symptom scores and FAST-PTSD scores, which is within the acceptable limits of binary logistic regressions. Analyses were conducted in SPSS v. 25. Statistical significance was determined at the 0.05 level and odds ratios (ORs) were used as the measure of effect size.

\section{Results}

At baseline, 243 women (81\%) reported clinically significant PTSD symptoms, and at 7 years, 71 women (26.2\%) did, indicating a significantly lower proportion of positive 
PTSD cases. A summary of the logistic regression model is outlined in Table 1 below. As shown, Block 1, the association between FAST-PTSD scores and clinically significant levels of PTSD at 7 years from baseline symptoms, was significant, $\chi^{2}(1)=4.77, p=0.029$, Nagelkerke $R^{2}=0.028$. Baseline PTSD symptoms were significantly associated with increased odds of clinically significant levels of PTSD at 7 years; however, the observed association was relatively weak $(\mathrm{OR}=1.201, p=0.036)$.

Block 2, including both baseline PTSD symptoms and FAST-PTSD scores, was also significant, $\chi^{2}(2)=12.28$, $p=0.002$, Nagelkerke $R^{2}=0.096$. When FAST-PTSD scores were included in the model, baseline PTSD scores were no longer significantly associated with clinically significant PTSD symptoms $(\mathrm{OR}=0.932, p=0.546)$. Baseline medium FASTPTSD scores were associated with nearly 2.5 greater odds of clinically significant PTSD at 7 years $(\mathrm{OR}=2.42, p=0.049)$, and women who reported baseline high FAST-PTSD risk scores were nearly eight times more likely to have clinically significant levels of PTSD at 7 years $(\mathrm{OR}=7.83, p=0.001)$.

\section{Discussion}

More than $25 \%$ of the women in the sample reported clinically significant PTSD symptoms 7 years after seeking help for IPV. This finding is consistent with the results of other prospective studies, indicating that many IPV victims suffer from significant mental health problems long after initially seeking help. ${ }^{4,6-7}$ The current study, however, is the first to follow women over a 7-year period. It is also noteworthy that more than $80 \%$ of the women reported clinically significant PTSD symptoms at baseline. Thus, many women who reported clinically significant PTSD symptoms when they first sought help for IPV did not report such problems 7 years later, but a substantial proportion did.

The results of the current study also indicated that the FAST-PTSD is significantly associated with clinical levels of PTSD symptoms 7 years after women first sought help for IPV. Moreover, FAST-PTSD scores were associated with future PTSD symptoms after accounting for baseline PTSD symptoms. In short, the FAST-PTSD appears to be a useful tool for service providers to help identify women at risk for clinically significant, long-term PTSD symptoms. Given the debilitating and far-reaching negative effects of PTSD, we view this as an extremely promising result. If women who are at risk for long-term PTSD can be successfully engaged in effective services for PTSD at the time they initially seek help, it may improve the long-term health outcomes for the women, as well as their dependent children.

It is important to emphasize that the outcome variable in the current study was simply a snapshot of clinically significant PTSD symptoms, measured 7 years after women first sought help for IPV. The women did not necessarily experience continuous PTSD symptoms over the entire 7-year period. In addition, the focus of the current study was on evaluating long-term associations between FAST-PTSD scores and clinically significant PTSD symptoms. It did not attempt to explain why some women experienced clinically significant PTSD symptoms, whereas others did not.

In addition to measuring PTSD symptoms, the FASTPTSD measures adverse childhood experiences, emotional support, and general self-efficacy, and it is certainly possible that one or more of these variables influenced the course of PTSD symptoms over time. However, this cannot be inferred directly from the results of the current study. Rather, future research will be necessary to elucidate reasons for the variability in women's experiences of PTSD following IPV.

A number of limitations to the current study should be acknowledged. The sample in the current study is the sample that was recruited for the initial development of the FASTPTSD. Thus, it will be important to replicate these findings in other samples. The sample consisted of women seeking help for IPV through the justice system or residential shelters. It is not clear whether the findings of this study would emerge for women in other clinical settings, such as outpatient clinics, hospitals, and rehabilitation centers. In addition, the current sample was restricted to women who were 18 years or older and who had a child. How FAST-PTSD scores relate to future PTSD symptoms for teenagers or women without children is not known. Finally, the current study was conducted in an urban location, and it is not clear how the findings might generalize to other nonurban locations. Despite these limitations, the FAST-PTSD appears to be a promising, userfriendly clinical screen for long-term PTSD following IPV.

\section{Conclusions}

Eighty-one percent of a cohort of 300 mothers who reached out for initial IPV services reported clinically significant symptoms of PTSD at baseline. Seven years later, $\sim 26 \%$ of the mothers reported clinically significant levels of PTSD. The 39-item FAST-PTSD, available as a web-based

Table 1. Summary of the Binary Logistic Regression

\begin{tabular}{|c|c|c|c|c|c|}
\hline & Beta & $S E$ & $O R$ & $\mathrm{p}$ & $95 \% C I$ \\
\hline \multicolumn{6}{|l|}{ Block 1} \\
\hline Constant & -2.054 & 0.496 & 0.128 & 0.000 & \\
\hline PTSD symptoms (base) & 0.184 & 0.087 & 1.201 & 0.036 & $1.012-1.426$ \\
\hline \multicolumn{6}{|l|}{ Block 2} \\
\hline Constant & -1.511 & 0.492 & 0.221 & 0.002 & \\
\hline PTSD symptoms (base) & -0.071 & 0.117 & 0.932 & 0.546 & $0.741-1.172$ \\
\hline Medium risk & 0.885 & 0.451 & 2.424 & 0.049 & $1.002-5.863$ \\
\hline High risk & 2.057 & 0.609 & 7.825 & 0.001 & $2.372-25.810$ \\
\hline
\end{tabular}

Model summary step 1: $\chi^{2}(1)=4.77, p=0.029$, Nagelkerke $R^{2}=0.028$; model summary step $2: \chi^{2}(2)=12.28, p=0.002$, Nagelkerke $R^{2}=0.096$.

CI, confidence interval; OR, odds ratio; PTSD, post-traumatic stress disorder; SE, standard error. 
application, was associated with the risk of clinically significant symptoms of PTSD 7 years after women's contact with IPV service providers. In short, the FAST-PTSD can offer service providers useful information to help inform clinical care decisions for women seeking services for IPV.

\section{Acknowledgments}

We are grateful to the clients, staff, and administrators of five shetlers in Harris County and to the clients, chief, and staff of the Harris County District Attorney's Office, Family Criminal Law Division.

\section{Author Disclosure Statement}

No competing financial interests exist.

\section{Funding Information}

Funding for this article was received from The Houston Endowment.

\section{References}

1. DeVries KM, Mak JY, Garcia-Moreno C, et al. The global prevalence of intimate partner violence against women. Science 2013;340:1527-1528.

2. Ehrensaft MK, Moffitt TE, Caspi A. Is domestic violence followed by an increased risk of psychiatric disorders among women but not among men? A longitudinal cohort study. Am J Psychiatry 2006;163:885-892.

3. Golding JM. Intimate partner violence as a risk factor for mental disorders. J Family Violence 1999;14:99-132.

4. Jouriles EN, McFarlane J, Vu NL, et al. Mothers' posttraumatic stress and child adjustment problems in families seeking services for intimate partner violence. J Consult Clin Psychol 2018;86:604-614.

5. Maddoux J, McFarlane J, Symes L, Fredland N, Feder G. Using baseline data to predict chronic PTSD 48-months after mothers report intimate partner violence: Outcomes for mothers and the intergenerational impact on child behavioral functioning. Arch Psychiatr Nurs 2018;32:475-482.

6. Johnson DM, Zlotnick, C. Remission of PTSD after victims of intimate partner violence leave a shelter. J Trauma Stress 2012;25:203-206.
7. Rivera EA, Sullivan CM, Zeoli AM, Bybee D. A longitudinal examination of mothers' depression and PTSD symptoms as impacted by partner-abusive men's harm to their children. J Interpers Violence 2018;33:2779-2801.

8. Kessler RC, Rose S, Koenen KC, et al. How well can posttraumatic stress disorder be predicted from pre-trauma risk factors? An exploratory study in the WHO world mental health surveys. World Psychiatry 2014;13:265274.

9. Resick PA, Williams LF, Suvak MK, Monson CM, Gradus JL. Long-term outcomes of cognitive-behavioral treatments for posttraumatic stress disorder among female rape survivors. J Consult Clin Psychol 2011;80:201-210.

10. Johnson DM, Zlotnick C, Perez SP, Johnson NL, Palmieri $\mathrm{P}$. Comparison of adding treatment of PTSD during and after shelter stay to standard care in residents of battered women's shelters: Results of a randomized clinical trial. J Trauma Stress 2016;29:365-373.

11. Symes L, Maddoux J, McFarlane J, Pennings J. A risk tool to predict sustained PTSD symptoms among women reporting abuse. J Womens Health 2016;25:340-347.

12. McFarlane J, Nava A, Gilroy H, Paulson R, Maddoux J. Testing two global models to prevent violence against women and children: Methods and policy implications for a seven year prospective study. Issues Ment Health Nurs 2012;33:871-881.

13. Breslau N, Peterson EL, Kessler RC Schultz LR. Short screening scale for DSM-IV posttraumatic stress disorder. Am J Psychiatry 1999;156:908-911.

14. Symes L, McFarlane J, Maddoux J, Levine L, Landrum KS, McFarlane CD. Establishing concurrent validity for a brief PTSD screen among women in a domestic violence shelter. J Interpers Violence 2018 [Epub ahead of print]; DOI: $10.1177 / 0886260518779595$.

Address correspondence to: Judith McFarlane, DrPH College of Nursing

Texas Woman's University 6700 Fannin

Houston, TX 77030

E-mail: jmcfarlane@twu.edu 